\title{
MODELING THE RELIABILITY INDICATORS' KINETICS OF A MOBILE ROPEWAY FORMED BY THE SELF-PROPELLED UNITS
}

\author{
Alexander Lagerev*, Igor Lagerev \\ Academician I.G. Petrovskii Bryansk State University, Bryansk, Russian Federation \\ *E-mail of corresponding author: avl-bstu@yandex.ru
}

\section{Resume}

A promising type of cargo vehicles are mobile ropeways formed by autonomous self-propelled rope units connected by a common cable transport system. The article presents a method for predicting the kinetics of the reliability indicators of the ropeway as a whole, individual rope units and their subsystems, taking into account the timing and volume of planned repairs. This method is advisable to use at the design stage of rope units, as it allows one to simulate the stage of their operation and provide the required level of reliability based on proactive replacement of structural elements that have reached the maximum permissible probability of critical failure. The method makes it possible to solve a number of important technical and economic problems related to operation of units and ropeways, including the development of an optimal strategy for their planned repairs, which has a minimum cost.

Available online: https://doi.org/10.26552/com.C.2022.2.B106-B119

\section{Article info}

Received 14 June 2021

Accepted 12 October 2021

Online 19 January 2022

\section{Keywords:}

mobile ropeway

self-propelled rope unit

probability-time analysis

kinetics of reliability indicators

rope system

repair

restoration

ISSN 1335-4205 (print version)

ISSN 2585-7878 (online version)

\section{Introduction}

Currently, mobile aerial ropeways should be considered as a promising type of overhead transport [1]. This is especially true in relation to the conditions of their use in unequipped and hard-to-reach territories with difficult terrain or unfavorable physical and mechanical properties of the surface soil. Such rope transport systems are effective for use in the ecosystems that are most vulnerable to negative anthropogenic impacts - forest, mountain alpine and Arctic territories [2-3]. Mobile ropeways also have advantages when it is necessary to provide the minimum terms of carrying out preparatory works for the commissioning of transport and transshipment technological operations $[1,4]$. Mobile rope transport systems are an important addition to stationary aerial ropeways, which have proven themselves as transport and logistics systems for cargo transportation and passenger traffic in urban and suburban conditions [5].

Mobile ropeways formed by autonomous selfpropelled units are intended for use in those places or in those conditions when it is impossible or not advisable to create capital structures (bridges, overpasses, tunnels, embankments, etc.) and therefore they can be used to quickly create crossings over water barriers, ravines, gorges, swampy areas, hotbeds of destruction or natural disasters. Mobile ropeways are most widely used in the forest and mining industries. Since the fifties of the last century [2], such rope systems have been successfully used in the forest industry in mountainous, heavily rugged or swampy areas for skidding extracted timber from the place of its direct development to the place of the entrance of trucks [6]. Currently, a number of machine-building enterprises (Valentini, Tajfun, Larix, Gantner, etc.) are present on the forestry equipment market, which serially produce the necessary technological equipment for gravity and pendulum ropeways with a wide range of technical characteristics [7-8]. In the mining industry, cargo ropeways are used to transport extracted raw materials to places of loading or processing [9]. It is also known to use mobile ropeways when performing construction and installation or repair work of autonomous objects, eliminating the consequences of natural or man-made disasters and performing agricultural work in mountainous areas.

It is advisable to create mobile ropeways based on the self-propelled wheeled or tracked chassis with high load capacity and cross-country ability. At the same time, the main technological equipment (including mechanisms for spatial orientation and movement of carrying-traction ropes, supporting structures and the mechanism for their installation and fixation in the working position) is placed on the load-bearing frames of self-propelled units [10]. This approach allows one to get the greatest autonomy of the entire rope transport system. 


\section{Setting a research problem}

The operation of stationary and mobile aerial ropeways is associated with the risk of occurrence and development of emergency situations that lead to a negative impact on the transported people or transported goods [6, 11]. Forecasting and analysis of the risk of such accidents in the operation of the main parts, systems or ropeways in general, as a rule, is based on application of the well-known method of fault trees [1213]. The main problem with the successful application of this method is that quantitative calculations of the failure probabilities of technical ropeways systems require knowledge of the individual failure probabilities of all the structural elements of these systems. Such information is extremely limited, since it is obtained by statistical processing of available data on failures that have occurred and does not take into account the duration of operation before failure [14]. As a result, the data necessary for predicting the risk of accidents on the probability of failure-free operation of structural elements are averaged and do not fully reflect the individual features of the design, modes and duration of operation of ropeways, as well as the effectiveness of the repair measures carried out [15].

In any case, the calculation of technical risk is based on a quantitative assessment of probability of the emergency events occurrence, [16]. This requires development of computational methods for predicting changes in reliability indicators over time and, first of all, the probabilities of critical failures during the operation of the technical object under study, taking into account the specific features of its design and operating modes [10].

\section{The studied construction and its mathematical model}

The studied design of self-propelled rope units on wheeled chassis of high load capacity and cross-country ability, which form a mobile single-span ropeway of the pendulum type, is shown in Figure 1. This design is protected by the patent [17] and is one of the promising modifications of the rope units based on a 4-axle chassis. The structural components and elements of the mechanism for installing and fixing the end tower in the working position are mounted directly on the load-bearing frame of the wheel chassis, including the end tower itself 1 and the lifting hydraulic cylinder 2. The end tower is a supporting structure for the nodes of the rope pulley orientation mechanism 3 and the carrying-traction rope movement mechanism 4 . The end tower and the lifting hydraulic cylinder are kinematically connected to each other and the loadbearing frame by the cylindrical hinges $A, B$ and $C$. The rope pulley orientation mechanism is necessary to coordinate the relative position of the rope pulleys of the coupled self-propelled rope units installed at different heights and to take into account the natural sagging of the carrying-traction rope [18]. The carrying-traction rope movement mechanism of one of the mobile units provides a pendulum movement of the transported goods along the route of the ropeway. The carryingtraction rope tension mechanism of another mobile unit ensures its optimal tension [4]. These mechanisms have a frequency-controlled hydraulic drive. Its operation is carried out by taking off the power of the own internal combustion engine of a self-propelled unit.

The main technological equipment of each selfpropelled rope unit, which ensures the operation of the mobile ropeway, consists of three structural subsystems [19]:

- the hydraulic subsystem providing the hydraulic drive of the main technological equipment, including the carrying-traction rope movement mechanism, the rope pulley orientation mechanism and the mechanism of installing and fixing of the end tower in the working position;

- the mechanical subsystem that provides the transmission and conversion of rotational motion and torque on the output shaft of the hydraulic motor of the carrying-traction rope movement mechanism into linear movement and traction force of the rope;

- the information and control subsystem that provides control over the operation of the rope system's structural elements, as well as the collection, processing, display and analysis in real time of the controlled quantitative parameters of the functional

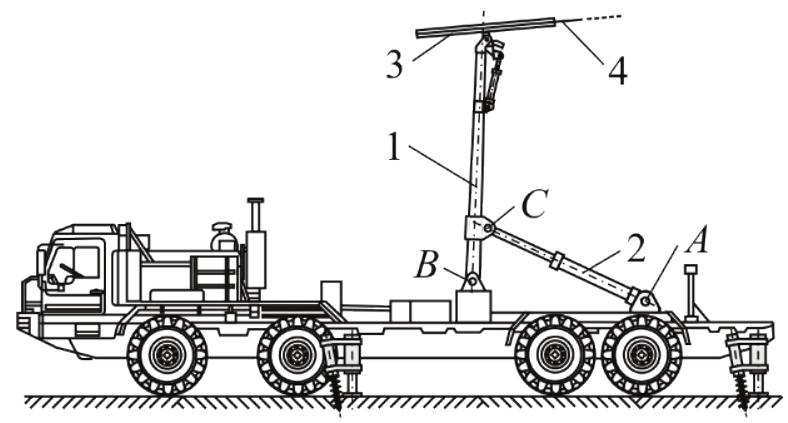

Figure 1 Appearance of a self-propelled rope unit in the working position:

1 - end tower; 2 - lifting hydraulic cylinder; 3 - rope pulley;

4 - carrying-traction rope; $A, B, C$ - cylindrical hinges 


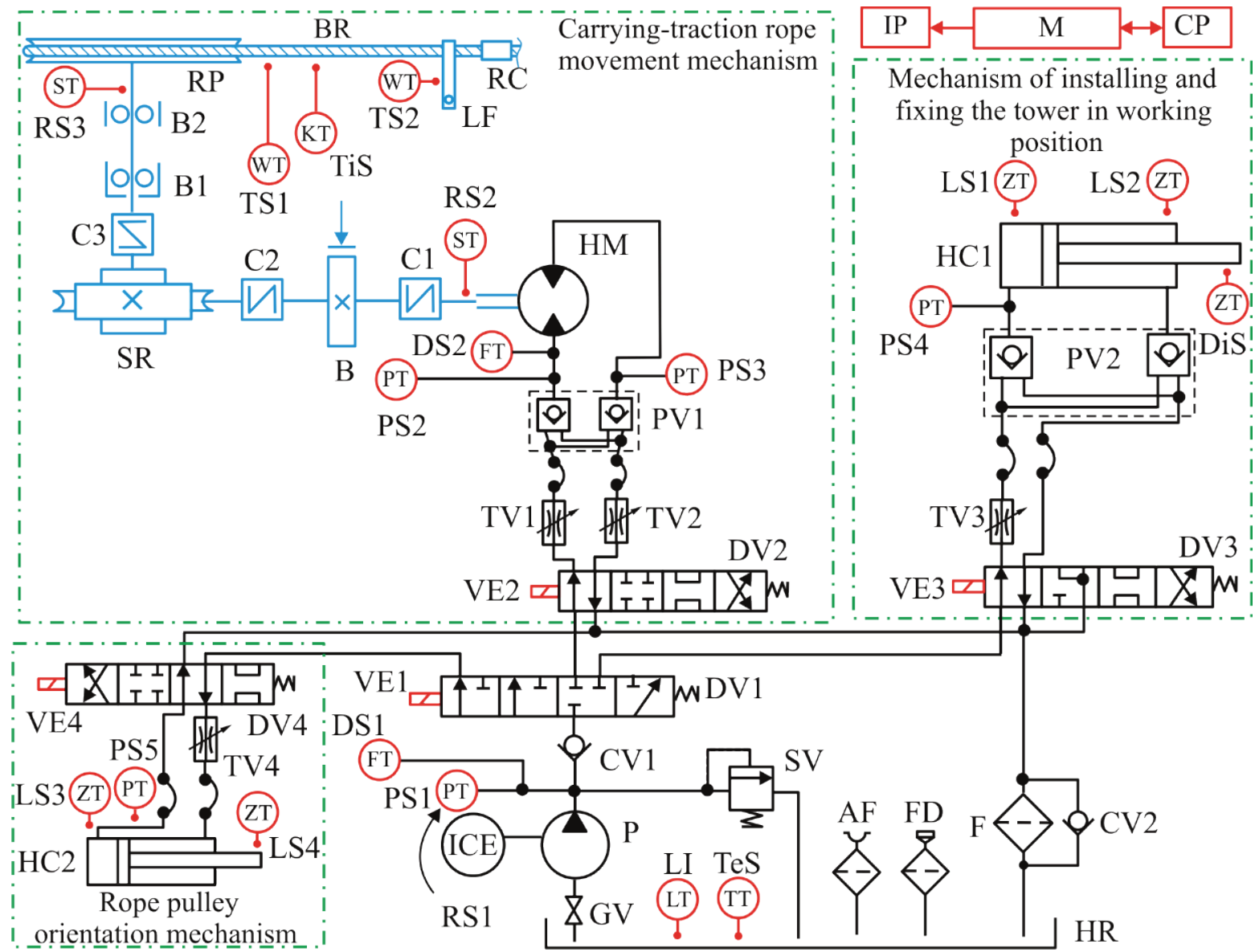

Figure 2 General block diagram of the main technological equipment of a self-propelled rope unit

state of the main technological equipment and the rope system, the formation of the necessary control actions.

In addition to these subsystems of both selfpropelled rope units, the structure of the mobile ropeway also includes a rope system that provides kinematic communication of self-propelled units and the cargo movement.

The structural elements of the chassis of the selfpropelled units are not included in the structure of the mobile ropeway, since their possible failures affect the reliability of the chassis themselves and are realized during the movement of units to the location of the ropeway. At this time, the technological equipment are are considered, servicing the operation of the ropeway, is in a non-working (transport) condition. On the contrary, during the operation of the mobile ropeway, the chassis is stationary. Thus, the time intervals of operation of the ropeway itself and the maintenance technological equipment do not coincide with the time intervals of operation of self-propelled chassis, which does not allow to combine the modeling of the reliability of these structures within a single mathematical model.

Figure 2 shows a general block diagram of the main technological equipment of a single self-propelled unit (Figure 1) and a rope system of mobile ropeway [19]. The scheme includes block diagrams of the specified subsystems. The following is a list of the structural elements of these subsystems and their numbering, which is used in the calculated dependencies of the mathematical model.

The hydraulic subsystem. The structural elements leading to critical failure: hydraulic pump $\mathrm{P}\left(h_{1,1}\right)$, hydraulic motor HM $\left(h_{12}\right)$, hydraulic cylinder of the mechanism of installing and fixing the end tower in the working position $\mathrm{HC} 1\left(h_{1,3}\right)$, hydraulic cylinder of the rope pulley orientation mechanism $\mathrm{HC} 2\left(h_{1,4}\right)$, hydraulic reservoir HR $\left(h_{1,5}\right)$, pressure safety valve $\mathrm{SV}\left(h_{1,6}\right)$, filter F $\left(h_{1,7}\right)$, gate directional control valve $\mathrm{DV} 1\left(h_{1,8}\right)$, directional control valve of the carrying-traction rope movement mechanism DV2 $\left(h_{19}\right)$, directional control valve of the mechanism of installing and fixing the end tower in the working position DV3 $\left(h_{1,10}\right)$, directional control valve of rope pulley orientation mechanism DV4 $\left(h_{1,11}\right)$, pilot operated check valve for fixing the rope pulley PV1 $\left(h_{1,12}\right)$, pilot operated check valve for fixing the end tower in working position PV2 $\left(h_{1,13}\right)$, metal lines $\left(h_{1,14}\right)$, flexible lines $\left(h_{1,15}\right)$, line connection devices (partially) $\left(h_{1,16}\right)$. The structural elements leading to non-critical failure: gate valve $\mathrm{GV}\left(h_{2,1}\right)$, check valve $\operatorname{CV} 1\left(h_{2,2}\right)$, check valve CV2 $\left(h_{2,3}\right)$, filling device FD $\left(h_{2,4}\right)$, air intake filter $\mathrm{AF}\left(h_{2,5}\right)$, throttle valves of the carrying-traction rope movement mechanism TV1, TV2 $\left(h_{2,6}\right)$, throttle valve of the mechanism for installing and fixing the end tower in the working position TV3 $\left(h_{27}\right)$, throttle valve for rope pulley orientation mechanism TV4 $\left(h_{2,8}\right)$, line connection 
devices (partially) $\left(h_{2,9}\right)$, hydraulic fluid $\left(h_{2,10}\right)$.

The mechanical subsystem. The structural elements leading to critical failure: rope pulley $\mathrm{RP}\left(m_{1,1}\right)$, speed reducer SR $\left(m_{1,2}\right)$, brake $\mathrm{B}\left(m_{1,3}\right)$, coupling $\mathrm{C} 1, \mathrm{C} 2, \mathrm{C} 3$ $\left(m_{1,4}\right)$, axial radial spherical roller bearing B1 $\left(m_{1,5}\right)$, radial spherical ball bearing $\mathrm{B} 2\left(m_{1,6}\right)$, transmission shafts $\left(m_{1,7}\right)$, carrying-traction rope $\mathrm{BR}\left(m_{1,8}\right)$, load handling fixture LF $\left(m_{1,9}\right)$, rope end connector RC $\left(m_{1,10}\right)$.

The information and control subsystem. The structural elements leading to critical failure: microprocessor $\mathrm{M}\left(e_{1,1}\right)$, control panel for rope system operation $\mathrm{CP}\left(e_{1,2}\right)$, information panel on the current state of the rope system IP $\left(e_{1,3}\right)$, gate directional control valve solenoid VE1 $\left(e_{1,4}\right)$, directional control valve solenoid of the carrying-traction rope movement mechanism VE2 $\left(e_{1,5}\right)$, directional control valve solenoid of the mechanism for installing and fixing the tower in the working position VE3 $\left(e_{1,6}\right)$, directional control valve solenoid of rope pulley orientation mechanism VE4 $\left(e_{1,7}\right)$, rotational velocity transmitter of the pump shaft RS1 $\left(e_{1,8}\right)$, rotational velocity transmitter of the rope pulley $\mathrm{RS} 3\left(e_{1,9}\right)$, tension transmitter of the carrying-traction rope TS1 $\left(e_{1,10}\right)$, weight transmitter of transported cargo TS2 $\left(e_{1,11}\right)$, transmitter for counting the time of the carrying-traction rope movement TiS $\left(e_{1,12}\right)$.The structural elements leading to non-critical failure: pump outlet pressure transmitter PS1 $\left(e_{2,1}\right)$, pressure transmitters in the hydraulic lines of the hydraulic motor PS2, PS3 $\left(e_{2,2}\right)$, pressure transmitter at the inlet of the hydraulic cylinder for installing the tower to the working position PS4 $\left(e_{2,3}\right)$, pressure transmitter at the inlet of the hydraulic cylinder of the rope pulley orientation PS5 $\left(e_{2,4}\right)$, pump volumetric flow transmitter $\operatorname{DS} 1\left(e_{2,5}\right)$, hydraulic motor volumetric flow transmitter DS2 $\left(e_{2,6}\right)$, rotational velocity transmitter of the hydraulic motor shaft RS2 $\left(e_{2,7}\right)$, end tower angle transmitter DiS $\left(e_{2,8}\right)$, limit switch for the lowest position of the tower LS1 $\left(e_{2,9}\right)$, limit switch for the upper end position of the tower LS2 $\left(e_{2,10}\right)$, limit switch for the lowest position of the rope pulley LS3 $\left(e_{2,11}\right)$, limit switch for the upper end position of the rope pulley LS4 $\left(e_{2,12}\right)$, hydraulic reservoir liquid level transmitter LI $\left(e_{2,13}\right)$, hydraulic reservoir fluid temperature transmitter TeS $\left(e_{2,14}\right)$.

The structural elements of the subsystems listed above are divided into two subgroups depending on the severity of the consequences of their failure on the performance of the rope system as a whole:

- elements leading to critical failure;

- elements that do not lead to critical failure.

A critical failure is a failure that results in the loss of the rope system operability (its transition to an inoperable state) and requires the immediate shutdown of the mobile ropeway. A non-critical failure is a failure that does not lead to a loss of the rope system operability, but only causes its transition to a faulty state, which does not require an immediate stop of the ropeway operation.

During its operation, a mobile ropeway formed by the two self-propelled rope units united by a single carrying-traction rope can be in one of three possible combinations of states:

- in good and operable states;

- in faulty and operable states;

- in faulty and inoperable states.

The first case fully corresponds to the regular operation of the mobile ropeway. In the second case, its operation is quite possible, although it is required to provide for the necessary recovery operations for those elements that had a non-critical failure by the time of the next planned breaks in operation. In the third case, the operation of the ropeway is impossible and requires an immediate stop and recovery operations, at least for the element that has a critical failure. Obviously, quantitative reliability indicators that characterize the transition of a mobile ropeway to the third combination of possible states should be used to assess the technical risk.

The main technological equipment of each selfpropelled unit consists of a significant number of elements that are potentially dangerous due to the possibility of their failure during the operation. The hydraulic subsystem includes $n_{h 1}=16$ elements leading to critical failure and $n_{h 2}=10$ elements leading to a non-critical failure. For a mechanical subsystem, the number of elements leading to a critical failure is $n_{m 1}$ $=7$. The information and control subsystem consists of $n_{e 1}=9$ elements leading to a critical failure and $n_{e 2}=14$ elements leading to a non-critical failure. Thus, for each self-propelled unit, the total number of elements leading to a critical failure is $N_{1}=32$ and the total number of elements leading to a non-critical failure is $N_{2}=24$.

For a mobile ropeway formed from two identical rope units and connected by a common rope system, the number of elements leading to failure will be greater. However, it should be borne in mind that not all the structural elements of both self-propelled units are used in the operation of the ropeway, so only one of these units should ensure the operation of the carrying-traction rope movement mechanism. The second unit is only necessary for turning the rope. Its carrying-traction rope movement mechanism during the operation of the ropeway does not work, the drive of the rope pulley is disabled and the rope pulley itself rotates freely due to contact interaction with the carrying-traction rope. Therefore, when analyzing the kinetics of the reliability indicators of a mobile ropeway, such structural elements of this rope unit (Figure 2) as HM, DV2, TV1, TV2, PV1 (in the hydraulic subsystem), SR, C1, C2, C3 (in the mechanical subsystem) and VE2, PS2, PS3, DS2, RS2, RS3 (in the information and control subsystem) should not be taken into account. The rope system includes $n_{m 1 r}=3$ and $n_{\text {e1r }}=3$ elements of the mechanical and information-control subsystems that lead to a critical failure. Thus, for a mobile ropeway, the total number of elements leading to critical failure is $N_{1}$ $=60$ and the total number of elements leading to noncritical failure is $N_{2}=42$. 


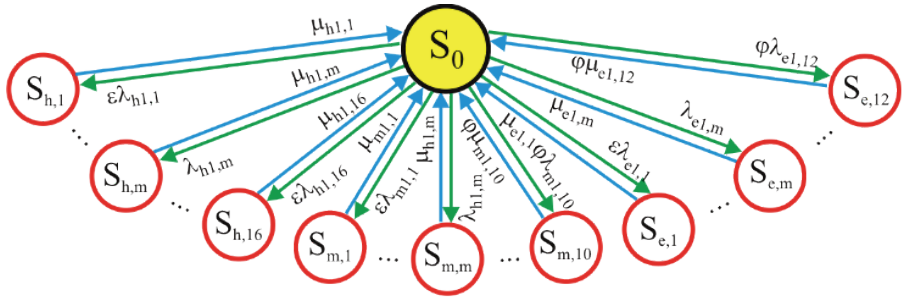

(a)

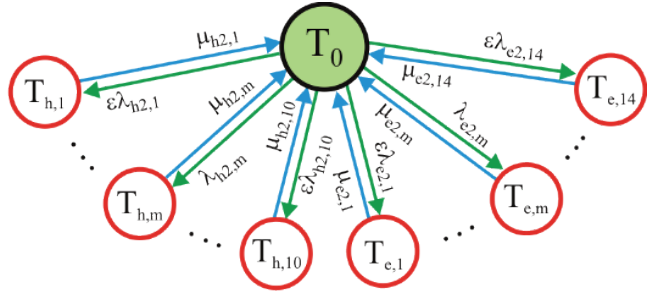

(b)

Figure 3 Graph of possible states and transitions between them during the operation of a technical item: a) in case of critical failures; $b$ ) in case of non-critical failures

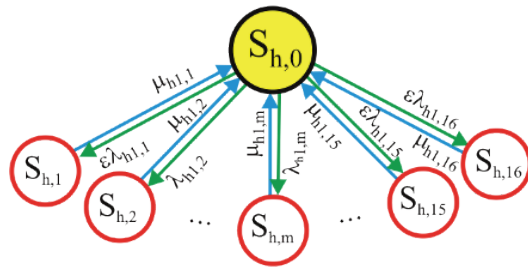

(a)

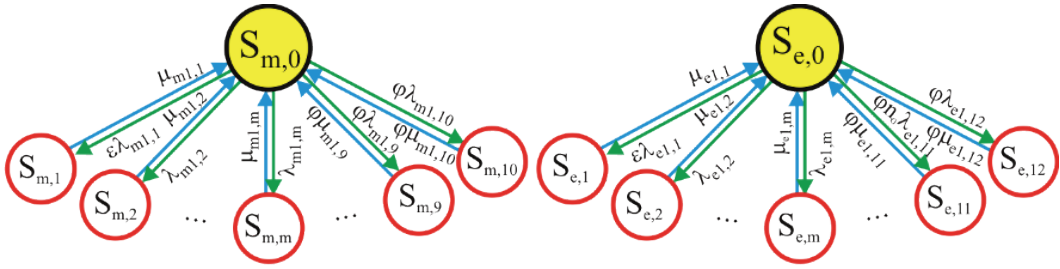

(b)

(c)

Figure 4 Graph of possible states and transitions between them during operation of periodically restored subsystems: a) hydraulic; b) mechanical (together with the rope system); c) information and control

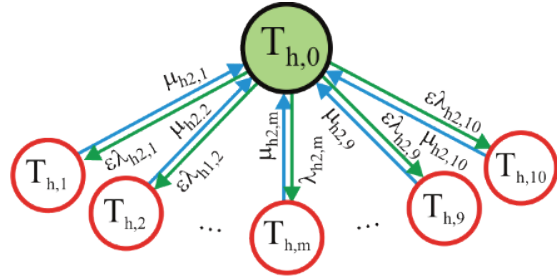

(a)

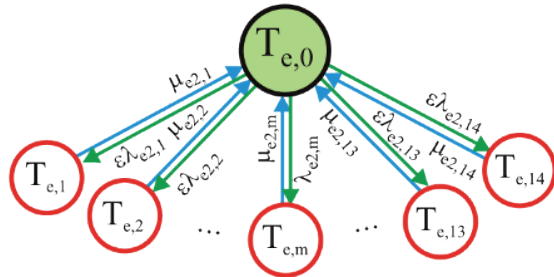

(b)

Figure 5 Graph of possible states and transitions between them during the operation of periodically repaired subsystems: a) hydraulic; b) information and control

The general approach to modeling the reliability kinetics and the structure of the corresponding calculation formulas, regardless of whether the technical item is considered - a separate self-propelled unit or a mobile ropeway as a whole. Therefore, the term "technical item under consideration" further refers to both of these items. The quantitative differences between them are taken into account using special coefficients in the calculation formulas.

When analyzing the probability of finding the technical item under consideration in an inoperable state during its operation, it should be taken into account that at any time it may be in one of the following possible states:

- one operable state $S_{0}$ (it is characterized by finding all the potentially dangerous elements in an operable state);

- one of several inoperable states $S_{1}, S_{2}, \ldots, S_{m}, \ldots$, $S_{N 1}$ (each state is characterized by finding one corresponding $m$-th element in an inoperable state when all the other $N_{1}-1$ elements are operable).

When analyzing the probability of finding the technical item under consideration in a faulty state during its operation, it should be taken into account that at any time it may be in one of the following possible states:
- $\quad$ one good state $T_{0}$ (it is characterized by the presence of all potentially dangerous elements in a good state and the compliance of the properties of the working fluid with the requirements of the operational documentation);

- one of several faulty states $T_{1}, T_{2}, \ldots, T_{m}, \ldots$, $T_{N 2}$ (each state is characterized by finding one corresponding $m$-th element in a faulty state when all the other $N_{2}-1$ elements are in a good state).

As an assumption, it is assumed that the probability of a subsequent failure of an arbitrary $q$-th element before the recovery of the failed $m$-th element is negligible. For the critical failures, this assumption is completely correct. For the non-critical failures, it is approximately correct, since in the event of such failures, the technical item can be operated for another period of time before the restoration are carried out during the planned break in the operation of the mobile ropeway.

Based on this assumption, the graphs of possible states and their connecting transitions during the operation of the technical item under consideration are constructed in Figure 3. They are a composition of separate subsystems similar in structure to the graphs - periodically restored subsystems (they form a graph in Figure 3a) and periodically repaired subsystems (they form a graph in Figure 3b). Graphs 
of individual subsystems are shown in Figures 4 and 5. The quantitative characteristics of the transition of the $v$-th subsystem from an operable state $S_{v, 0}$ to an inoperable state $S_{v m}$, caused by the failure of the $m$-th element, is the failure rate $\lambda_{v 1, m}$ of this element. The quantitative characteristics of the reverse transition from an inoperable state $S_{v, m}$ to an operable state $S_{v, 0}$ caused by the restoration or replacement of a previously failed $m$-th element, is the restoration rate $\mu_{v 1, m}$ of this element. Similarly, the transitions from a good state $T_{v, 0}$ to a faulty state $T_{v, m}$ are quantitatively characterized the failure rate $\lambda_{v 2, m}{ }^{v, m}$ and the restoration rate $\mu_{v 2, m}$. The coefficients $\varphi$ and $\varepsilon$ depend on the technical item under consideration: for a separate self-propelled rope unit $\varphi=0$ and $\varepsilon=1$, for a mobile ropeway $\varphi=1$ and $\varepsilon=2$.

The probabilities $P_{0}, P_{1}, P_{2}, \ldots, P_{m}, \ldots, P_{N 1}$ (or $U_{0}, U_{1}$, $U_{2}, \ldots, U_{m}, \ldots, U_{N 2}$ ) of finding the technical item under consideration at an arbitrary time of operation in all the possible states $S_{0}, S_{1}, S_{2}, \ldots, S_{m}, \ldots, S_{N 1}$ (or $T_{0}, T_{1}, T_{2}$, $\ldots, T_{m}, \ldots, T_{N 2}$ ) can be determined using the ChapmanKolmogorov equation [14]. For the graphs in Figures $3-5$, it is expressed by a system of ordinary differential equations of the first order.

When analyzing the transition of a technical item from an operable state to an inoperable state (graph in Figure 3a), the system of equations will have the following form:

$$
\left\{\begin{array}{c}
\dot{P}_{0} \\
\left\{\dot{P}_{h}\right\} \\
\left\{\dot{P}_{m}\right\} \\
\left\{\dot{P}_{e}\right\}
\end{array}\right\}=\left[\begin{array}{cccc}
-\Lambda_{1} & \left\{\omega_{h \mu}\right\} & \left\{\omega_{m \mu}\right\} & \left\{\omega_{e \mu}\right\} \\
\left\{\omega_{h \lambda}\right\} & {\left[\Omega_{h h}\right]} & {[0]_{h m}} & {[0]_{h e}} \\
\left\{\omega_{m \lambda}\right\} & {[0]_{h m}^{\prime}} & {\left[\Omega_{m m}\right]} & {[0]_{m e}} \\
\left\{\omega_{e \lambda}\right\} & {[0]_{h e}^{\prime}} & {[0]_{m e}^{\prime}} & {\left[\Omega_{e e}\right]}
\end{array}\right] \cdot\left\{\begin{array}{c}
\dot{P}_{0} \\
\left\{\dot{P}_{h}\right\} \\
\left\{\dot{P}_{m}\right\} \\
\left\{\dot{P}_{e}\right\}
\end{array}\right\},
$$

where:

$$
\begin{aligned}
& \{P\}^{\prime}=\left\{P_{0}\left\{P_{h}\right\}\left\{P_{m}\right\}\left\{P_{e}\right\}\right\}=\left\{P_{0}\left\{P_{h, 1} \ldots P_{h, 16}\right\}\right. \\
& \left\{P_{m, 1} \ldots P_{m, 10}\right\}\left\{P_{e, 1} \ldots P_{e, 12}\right\} ; \\
& \{\dot{P}\}^{\prime}=\left\{\dot{P}_{0}\left\{\dot{P}_{h}\right\}\left\{\dot{P}_{m}\right\}\left\{\dot{P}_{e}\right\}\right\}=\left\{\dot{P}_{0}\left\{\dot{P}_{h, 1} \ldots \dot{P}_{h, 16}\right\}\right. \\
& \left\{\dot{P}_{m, 1} \ldots \dot{P}_{m, 10}\right\}\left\{\dot{P}_{e, 1} \ldots \dot{P}_{e, 12}\right\} ; \\
& \Lambda_{1}=\Lambda_{h 1}+\Lambda_{m 1}+\Lambda_{e l} ; \Lambda_{h 1}=\varepsilon \sum_{m=1}^{m=16} \lambda_{h 1, m}- \\
& (\varepsilon-1)\left(\lambda_{h 1,2}+\lambda_{h 1,9}\right) ; \\
& \Lambda_{m 1}=\varepsilon\left(\sum_{m=1}^{m=7} \lambda_{m 1, m}+2 \lambda_{m 1,4}\right)-(\varepsilon-1) . \\
& \cdot\left(\lambda_{m 1,2}+3 \lambda_{m 1,4}\right)+\varphi\left(\lambda_{m 1,8}+n_{G} \lambda_{m 1,9}+\lambda_{m 1,10}\right) ; \\
& \Lambda_{e 1}=\varepsilon \sum_{m=1}^{m=9} \lambda_{e 1, m}-(\varepsilon-1)\left(\lambda_{e 1,5}+\lambda_{e 1,9}\right)+\varphi\left(\lambda_{e 1,10}+\right. \\
& \left.+n_{G} \lambda_{e 1,11}+\lambda_{m 1,10}\right) ; \\
& \left\{\omega_{h \mu}\right\}=\left\{\mu_{h 1,1} \mu_{h 1,2} \mu_{h 1,3} \mu_{h 1,4} \mu_{h 1,5} \mu_{h 1,6} \mu_{h 1,7} \mu_{h 1,8} \mu_{h 1,9} \mu_{h 1,10}\right. \\
& \left.\mu_{h 1,11} \mu_{h 1,12} \mu_{h 1,13} \mu_{h 1,14} \mu_{h 1,15} \mu_{h 1,16}\right\} ; \\
& \left\{\omega_{m \mu}\right\}=\left\{\mu_{m 1,1} \mu_{m 1,2} \mu_{m 1,3} \mu_{m 1,4} \mu_{m 1,5} \mu_{m 1,6} \mu_{m 1,7} \mu_{m 1,8}\right. \\
& \left.\mu_{m 1,9} \mu_{m 1,10}\right\} ; \\
& \left\{\omega_{e \mu}\right\}=\left\{\mu_{e 1,1} \mu_{e 1,2} \mu_{e 1,3} \mu_{e 1,4} \mu_{e 1,5} \mu_{e 1,6} \mu_{e 1,7} \mu_{e 1,8} \mu_{e 1,9} \mu_{e 1,10}\right. \\
& \left.\mu_{e 1,11} \mu_{e 1,12}\right\} ; \\
& \left\{\omega_{h \lambda}\right\}^{\prime}=\left\{\varepsilon \lambda_{h 1,1} \lambda_{h 1,2} \varepsilon \lambda_{h 1,3} \varepsilon \lambda_{h 1,4} \varepsilon \lambda_{h 1,5} \varepsilon \lambda_{h 1,6} \varepsilon \lambda_{h 1,7} \varepsilon \lambda_{h 1,8}\right. \\
& \left.\mu \varepsilon \lambda_{h 1,9} \varepsilon \lambda_{h 1,10} \varepsilon \lambda_{h 1,11} \varepsilon \lambda_{h 1,12} \varepsilon \lambda_{h 1,13} \varepsilon \lambda_{h 1,14} \varepsilon \lambda_{h 1,15} \varepsilon \lambda_{h 1,16}\right\} ;
\end{aligned}
$$

$\left\{\omega_{m \mu}\right\}^{\prime}=\left\{\varepsilon \lambda_{m 1,1} \lambda_{m 1,2} \varepsilon \lambda_{m 1,3} \varepsilon \lambda_{m 1,4} \varepsilon \lambda_{m 1,5} \varepsilon \lambda_{m 1,6} \varepsilon \lambda_{m 1,7}\right.$ $\left.\varepsilon \lambda_{m 1,8} \varepsilon \lambda_{m 1,9} \varepsilon \lambda_{m 1,10}\right\}$;

$\left\{\omega_{e \lambda}\right\}^{\prime}=\left\{\varepsilon \lambda_{e 1,1} \varepsilon \lambda_{e 1,2} \varepsilon \lambda_{e 1,3} \varepsilon \lambda_{e 1,4} \varepsilon \lambda_{e 1,5} \varepsilon \lambda_{e 1,6} \varepsilon \lambda_{e 1,7} \varepsilon \lambda_{e 1,8}\right.$ $\left.\varepsilon \lambda_{e 1,9} \varepsilon \lambda_{e 1,10} \varepsilon \lambda_{e 1,11} \varepsilon \lambda_{e 1,12}\right\}$;

$[\mathbf{0}]_{h m},[\mathbf{0}]_{h e},[\mathbf{0}]_{m e}$ are the null matrices having the sizes $10 \times 16,12 \times 16,12 \times 10 ;\left[\Omega_{h h}\right],\left[\Omega_{m m}\right],\left[\Omega_{e e}\right]$ are the diagonal matrices of the form

$\operatorname{diag}\left[\Omega_{h h}\right]=\left\{-\mu_{h 1,1}-\mu_{h 1,2}-\mu_{h 1,3}-\mu_{h 1,4}-\mu_{h 1,5}\right.$

$-\mu_{h 1,6}-\mu_{h 1,7}-\mu_{h 1,8}-\mu_{h 1,9}-\mu_{h 1,10}-\mu_{h 1,11}-\mu_{h 1,12}$

$\left.-\mu_{h 1,13}-\mu_{h 1,14}-\mu_{h 1,15}-\mu_{h 1,16}\right\}$;

$\operatorname{diag}\left[\Omega_{m m}\right]=\left\{-\mu_{m 1,1}-\mu_{m 1,2}-\mu_{m 1,3}-\mu_{m 1,4}-\mu_{m 1,5}\right.$

; $\left.-\mu_{m 1,6}-\mu_{m 1,7}-\mu_{m 1,8}-\mu_{m 1,9}-\mu_{m 1,10}\right\}$;

$\operatorname{diag}\left[\Omega_{e e}\right]=\left\{-\mu_{e 1,1}-\mu_{e 1,2}-\mu_{e 1,3}-\mu_{e 1,4}-\mu_{e 1,5}\right.$

$\left.-\mu_{e 1,6}-\mu_{e 1,7}-\mu_{e 1,8}-\mu_{e 1,9}-\mu_{e 1,10}-\mu_{e 1,11}-\mu_{e 1,12}\right\}$;

$\{\ldots\}^{\prime}$ is transpose operation.

For individual subsystems (graphs in Figure 4) their transition from an operable state to an inoperable state will be expressed by systems of equations of the following form: for the hydraulic subsystem

$\left\{\begin{array}{c}\dot{P}_{h, 0} \\ \left\{\dot{P}_{h}\right\}\end{array}\right\}=\left[\begin{array}{cc}-\Lambda_{h 1} & \left\{\omega_{h \mu}\right\} \\ \left\{\omega_{h \lambda}\right\} & {\left[\Omega_{h h}\right.}\end{array}\right] \cdot\left\{\begin{array}{c}P_{h, 0} \\ \left\{P_{h}\right\}\end{array}\right\}$

for the mechanical subsystem

$\left\{\begin{array}{c}\dot{P}_{h, 0} \\ \left\{\dot{P}_{m}\right\}\end{array}\right\}=\left[\begin{array}{cc}-\Lambda_{m 1} & \left\{\omega_{m \mu}\right\} \\ \left\{\omega_{m \lambda}\right\} & {\left[\Omega_{m m}\right.}\end{array}\right] \cdot\left\{\begin{array}{c}P_{m, 0} \\ \left\{P_{m}\right\}\end{array}\right\} ;$

for the information and control subsystem

$\left\{\begin{array}{c}\dot{P}_{h, 0} \\ \left\{\dot{P}_{e}\right\}\end{array}\right\}=\left[\begin{array}{cc}-\Lambda_{e 1} & \left\{\omega_{e \mu}\right\} \\ \left\{\omega_{e \lambda}\right\} & {\left[\Omega_{e e}\right]}\end{array}\right] \cdot\left\{\begin{array}{c}P_{e, 0} \\ \left\{P_{e}\right\}\end{array}\right\}$.

When analyzing the transition of a technical item from a good state to a faulty state (graph in Figure 3b), the system of equations will have the following form

$$
\left\{\begin{array}{c}
\dot{U}_{0} \\
\left\{\dot{U}_{h}\right\} \\
\left\{\dot{U}_{e}\right\}
\end{array}\right\}=\left[\begin{array}{ccc}
-\Lambda_{2} & \left\{\psi_{h \mu}\right\} & \left\{\psi_{e \mu}\right\} \\
\left\{\psi_{h \lambda}\right\} & {\left[\Psi_{h h}\right]} & {[0]_{e h}} \\
\left\{\psi_{e \lambda}\right\} & {[0]_{e h}^{\prime}} & {\left[\Psi_{e e}\right]}
\end{array}\right] \cdot\left\{\begin{array}{c}
U_{0} \\
\left\{U_{h}\right\} \\
\left\{U_{e}\right\}
\end{array}\right\},
$$

where:

$$
\begin{aligned}
& \{U\}^{\prime}=\left\{U_{0}\left\{U_{h}\right\}\left\{U_{e}\right\}\right\}=\left\{U_{0}\left\{U_{h, 1} \ldots U_{h, 10}\right\}\right. \\
& \left.\left\{U_{e, 1} \ldots U_{e, 14}\right\}\right\} ; \\
& \{\dot{U}\}^{\prime}=\left\{\dot{U}_{0}\left\{\dot{U}_{h}\right\}\left\{\dot{U}_{e}\right\}\right\}=\left\{\dot{U}_{0}\left\{\dot{U}_{h, 1} \ldots \dot{U}_{h, 10}\right\}\right. \\
& \left.\left\{\dot{U}_{e, 1} \ldots \dot{U}_{e, 14}\right\}\right\} ; \Lambda_{2}=\Lambda_{h 2}+\Lambda_{e 2} ; \\
& \Lambda_{h 2}=\varepsilon \sum_{m=1}^{m=10} \lambda_{h 2, m}+(2-\varepsilon) \lambda_{h 2,6} ; \\
& \Lambda_{e 2}=\varepsilon \sum_{m=1}^{m=14} \lambda_{e 2, m}+(2-\varepsilon) \lambda_{e 2,2}-(\varepsilon-1)\left(\lambda_{e 2,6}+{ }_{e 2,7}\right) ; \\
& \left\{\Psi_{h \mu}\right\}=\left\{\mu_{h 2,1} \mu_{h 2,2} \mu_{h 2,3} \mu_{h 2,4} \mu_{h 2,5} \mu_{h 2,6} \mu_{h 2,7} \mu_{h 2,8} \mu_{h 2,9}\right. \\
& \left.\mu_{h 2,10}\right\} ; \\
& \left\{\Psi_{e \mu}\right\}=\left\{\mu_{e 2,1} \mu_{e 2,2} \mu_{e 2,3} \mu_{e 2,4} \mu_{e 2,5} \mu_{e 2,6} \mu_{e 2,7} \mu_{e 2,8} \mu_{e 2,9} \mu_{e 2,10}\right. \\
& \left.\mu_{e 2,11} \mu_{e 2,12} \mu_{e 2,13} \mu_{e 2,14}\right\} ; \\
& \left\{\Psi_{h \lambda}\right\}^{\prime}=\left\{\varepsilon \lambda_{h 2,1} \varepsilon \lambda_{h 2,2} \varepsilon \lambda_{h 2,3} \varepsilon \lambda_{h 2,4} \varepsilon \lambda_{h 2,5} \varepsilon \lambda_{h 2,6} \varepsilon \lambda_{h 2,7} \varepsilon \lambda_{h 2,8}\right. \\
& \left.\varepsilon \lambda_{h 2,9} \varepsilon \lambda_{h 2,10}\right\} ; \\
& \left\{\Psi_{e \lambda}\right\}^{\prime}=\left\{\varepsilon \lambda_{e 2,1} \varepsilon \lambda_{e 2,2} \varepsilon \lambda_{e 2,3} \varepsilon \lambda_{e 2,4} \varepsilon \lambda_{e 2,5} \varepsilon \lambda_{e 2,6} \varepsilon \lambda_{e 2,7} \varepsilon \lambda_{e 2,8}\right. \\
& \left.\varepsilon \lambda_{e 2,9} \varepsilon \lambda_{e 2,10} \varepsilon \lambda_{e 2,11} \varepsilon \lambda_{e 2,12} \varepsilon \lambda_{e 2,13} \varepsilon \lambda_{e 2,14}\right\} ;
\end{aligned}
$$


$[0]_{e h}$ is the null matrix having the size $14 \times 10 ;\left[\Psi_{h h}\right],\left[\Psi_{\rho e}\right]$ are the diagonal matrices of the form

$\operatorname{diag}\left[\Psi_{e e}\right]=\left\{-\mu_{e 2,1}-\mu_{e 2,2}-\mu_{e 2,3}-\mu_{e 2,4}-\mu_{e 2,5}\right.$

$-\mu_{e 2,6}-\mu_{e 2,7}-\mu_{e 2,8}-\mu_{e 2,9}-\mu_{e 2,10}-\mu_{e 2,11}-\mu_{e 2,12}$

$\left.-\mu_{e 2,13}-\mu_{e 2,14}\right\}$

$\operatorname{diag}\left[\Psi_{h h}\right]=\left\{-\mu_{h 2,1}-\mu_{h 2,2}-\mu_{h 2,3}-\mu_{h 2,4}-\mu_{h 2,5}\right.$

$\left.-\mu_{h 2,6}-\mu_{h 2,7}-\mu_{h 2,8}-\mu_{h 2,9}-\mu_{h 2,10}\right\}$.

For individual subsystems (graphs in Figure 5) their transition from a good state to a faulty state will be expressed by systems of equations of the following form: for the hydraulic subsystem

$$
\left\{\begin{array}{c}
\dot{U}_{h, 0} \\
\left\{\dot{U}_{h}\right\}
\end{array}\right\}=\left[\begin{array}{cc}
-\Lambda_{h 2} & \left\{\psi_{h \mu}\right\} \\
\left\{\psi_{h \lambda}\right\} & {\left[\Psi_{h h}\right.}
\end{array}\right] \cdot\left\{\begin{array}{c}
U_{h, 0} \\
\left\{U_{h}\right\}
\end{array}\right\}
$$

for the information and control subsystem

$$
\left\{\begin{array}{c}
\dot{U}_{e, 0} \\
\left\{\dot{U}_{e}\right\}
\end{array}\right\}=\left[\begin{array}{cc}
-\Lambda_{e 2} & \left\{\psi_{e \mu}\right\} \\
\left\{\psi_{e \lambda}\right\} & {\left[\Psi_{e e}\right]}
\end{array}\right] \cdot\left\{\begin{array}{c}
U_{e, 0} \\
\left\{U_{e}\right\}
\end{array}\right\} \text {. }
$$

The initial conditions for solving systems of differential Equations (1) - (7) include a set of probability values $P_{m}$ or $U_{m}$ at the time of putting the technical object and its subsystems into operation (at $\tau=\tau_{0}=0$ ). Therefore, the vectors of the initial conditions have the following form

$$
\begin{aligned}
& \{P(0)\}^{\prime}=\left\{P_{0}(0)\left\{P_{h, 1}(0) \ldots P_{h, 16}(0)\right\}\right. \\
& \left.\left\{P_{m, 1}(0) \ldots P_{m, 10}(0)\right\}\left\{P_{e, 1}(0) \ldots P_{e, 12}(0)\right\}\right\}= \\
& =\{1\{0 \ldots 0\}\{0 \ldots 0\}\{0 \ldots 0\}\} \\
& \{U(0)\}^{\prime}=\left\{U_{0}(0)\left\{U_{h, 1}(0) \ldots U_{h, 10}(0)\right\}\right. \\
& \left.\left\{U_{e, 1}(0) \ldots U_{e, 14}(0)\right\}\right\}= \\
& =\{1\{0 \ldots 0\}\{0 \ldots 0\}\{0 \ldots 0\}\} ; \\
& \left\{P_{h, 0}(0)\left\{P_{h}(0)\right\}\right\}^{\prime}= \\
& \left\{P_{h, 0}(0)\left\{P_{h, 1}(0) \ldots P_{h, 16}(0)\right\}\right\}=\{1\{0 \ldots 0\}\} ; \\
& \left\{U_{h, 0}(0)\left\{U_{h}(0)\right\}\right\}^{\prime}= \\
& \left\{U_{h, 0}(0)\left\{U_{h, 1}(0) \ldots U_{h, 10}(0)\right\}\right\}=\{1\{0 \ldots 0\}\} ; \\
& \left\{P_{m, 0}(0)\left\{P_{m}(0)\right\}\right\}^{\prime}= \\
& \left\{P_{m, 0}(0)\left\{P_{m, 1}(0) \ldots P_{m, 10}(0)\right\}\right\}=\{1\{0 \ldots 0\}\} ; \\
& \left\{P_{e, 0}(0)\left\{P_{e}(0)\right\}\right\}^{\prime}= \\
& \left\{P_{e, 0}(0)\left\{P_{e, 1}(0) \ldots P_{e, 12}(0)\right\}\right\}=\{1\{0 \ldots 0\}\} ; \\
& \left\{U_{e, 0}(0)\left\{U_{e}(0)\right\}\right\}^{\prime}= \\
& \left\{U_{e, 0}(0)\left\{U_{e, 1}(0) \ldots U_{e, 14}(0)\right\}\right\}=\{1\{0 \ldots 0\}\} .
\end{aligned}
$$

The systems of Equations in (1) - (4) allow to predict the process of changing the probability of failurefree operation of a technical item $P_{r s}(\tau)$ and its $v$-th subsystem $P_{v}(\tau)$ over time, since

$$
\begin{aligned}
& P_{r s}(\tau)=P_{0}(\tau) ; P_{h}(\tau)=P_{h, 0}(\tau) ; \\
& P_{m}(\tau)=P_{m, 0}(\tau) ; P_{e}(\tau)=P_{e, 0}(\tau) .
\end{aligned}
$$

The sum of all the other probabilities expresses the probability of finding the technical item $Q_{r s}(\tau)$ and the $v$-th subsystem $Q_{v}(\tau)$ in an inoperable state:

$$
\begin{aligned}
& Q_{r s}(\tau)=1-P_{r s}(\tau)=\sum_{m=1}^{m=n_{h 1}} P_{h, m}(\tau)+ \\
& +\sum_{m=1}^{m=n m 1} P_{m, m}(\tau)+\sum_{m=1}^{m=n_{e 1}} P_{e, m}(\tau) ;
\end{aligned}
$$

$Q_{h}(\tau)=1-P_{h}(\tau)=\sum_{m=1}^{m=n h 1} P_{h, m}(\tau)$

$Q_{m}(\tau)=1-P_{m}(\tau)=\sum_{m=1}^{m=n m 1} P_{m, m}(\tau)$

$Q_{e}(\tau)=1-P_{e}(\tau)=\sum_{m=1}^{m=n_{e 1}} P_{e, m}(\tau)$.

The ratio $P_{m}(\tau) / Q_{r s}(\tau)$ can be considered as the relative individual contribution of the critical failure of the $m$-th element of the technical item to the decrease in the overall level of its reliability at an arbitrary time $\tau$. Accordingly, the ratio $P_{v, m}(\tau) / Q_{v}(\tau)$ can be considered as the relative individual contribution of the critical failure of the $m$-th element of the $v$-th subsystem to the decrease in the overall level of reliability of this subsystem at an arbitrary time $\tau$.

The systems of Equations (5) - (7) allow to predict the kinetics of the probability of good operation of a technical item $U_{r s}(\tau)$ and its $v$-th subsystem $U_{v}(\tau)$, since

$$
\begin{aligned}
& U_{r s}(\tau)=U_{0}(\tau) ; U_{h}(\tau)=U_{h, 0}(\tau) ; \\
& U_{e}(\tau)=U_{e, 0}(\tau) .
\end{aligned}
$$

The sum of all the other probabilities expresses the probability of finding a technical item $V_{r s}(\tau)$ and its $v$-th subsystem $V_{v}(\tau)$ in a faulty state:

$$
\begin{aligned}
& V_{r s}(\tau)=1-U_{r s}(\tau)=\sum_{m=1}^{m=n h 2} U_{h, m}(\tau)+ \\
& +\sum_{m=1}^{m=n_{e 2}} U_{e, m}(\tau) ;
\end{aligned}
$$

$V_{h}(\tau)=1-U_{h}(\tau)=\sum_{m=1}^{m=n h 2} U_{h, m}(\tau)$

$$
V_{e}(\tau)=1-U_{e}(\tau)=\sum_{m=1}^{m=n_{e 2}} U_{e, m}(\tau)
$$

The solution of systems in Equations (1) - (7) under initial conditions in Equations (8) - (14) adequately characterizes the change in reliability indicators over time until the first scheduled repair $\tau_{r, 1}$. The moments of scheduled repairs $\tau_{r n}$ are established at the design stage of self-propelled rope units. They are associated with the repair of such structural elements that do not lead to a critical failure, do not entail an immediate stop of the operation of the ropeway and do not require restoration work.

Figure 6 shows a qualitative graph of the time 


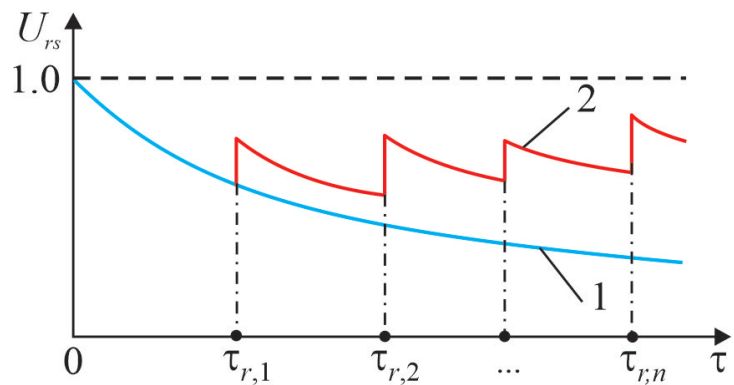

Figure 6 Kinetics of probability of finding a technical item in a good state: 1 - without repairs; 2 - with repairs

variation of the probability of finding a technical object $U_{r s}(\tau)$ and its subsystems in a good state during the periodic repairs and in their absence.

At a given time $\tau_{r, 1}$, one or more structural elements are repaired. Therefore, the probability of finding an arbitrary $m$-th element of the $v$-th subsystem in a faulty state abruptly decreases from the value $U_{v, m}\left(\tau_{r, 1}-0\right)=$ $U_{v, m}\left(\tau_{r, 1}\right)$ to the value $U_{v, m}\left(\tau_{r, 1}+0\right)=0$. The probability of finding a technical item and its subsystems in good state increases abruptly from $U_{0}\left(\tau_{r, 1}-0\right)=U_{0}\left(\tau_{r, 1}\right)$ by the sum of the probabilities of the repaired elements $U_{v, m}\left(\tau_{r, 1}\right)$. Therefore, from the moment of time, the integration of systems of differential Equations (5) (7) should be carried out with new initial conditions vectors. The first elements of these new vectors take the following values:

$$
\begin{aligned}
& U_{0}\left(\tau_{r, 1}+0\right)=U_{0}\left(\tau_{r, 1}\right)+\sum_{m=1}^{m=n h 2, r, 1} U_{h, m}\left(\tau_{r, 1}\right)+ \\
& +\sum_{m=1}^{m=n_{e 2}, r, 1} U_{e, m}\left(\tau_{r, 1}\right) ; \\
& U_{h, 0}\left(\tau_{r, 1}+0\right)=U_{h, 0}\left(\tau_{r, 1}\right)+\sum_{m=1}^{m=n_{h 2, r, 1}} U_{h, m}\left(\tau_{r, 1}\right) ; \\
& U_{e, 0}\left(\tau_{r, 1}+0\right)=U_{e, 0}\left(\tau_{r, 1}\right)+\sum_{m=1}^{m=n_{e 2, r, 1}} U_{e, m}\left(\tau_{r, 1}\right) ;
\end{aligned}
$$

The elements of the new initial condition vectors that correspond to the repaired structural elements of the subsystems are reset to zero and the remaining elements retain their values.

The need to repair the $m$-th element of the $v$-th subsystem during the $i$-th scheduled repair $\tau_{r i}$ is determined by the periodicity of its repairs $\Delta T_{v, m}$. This periodicity is set by the operational documentation.

Similarly, the vectors of the initial conditions in Equation (22) and (23) change for other time moments $\tau_{r, n}$. Thus, the process of predicting changes in the reliability indicators of the technical item under consideration and its subsystems, which determine their presence in a good or faulty state, during the scheduled repair activities of elements after a non-critical failure (curve 2 in Figure 6) is reduced to the sequential integration of systems of differential Equations (1) - (7) within sequentially arranged time intervals $\tau_{r, n} \leq \tau \leq$ $\tau_{r, n+1}$. The vectors of the initial conditions in Equations
(22) and (23) at the starting moment of each such interval $\tau_{r, n}$ are subject to periodic reformation.

Experience in the operation of transport and transshipment machines and equipment of various types [20] shows that, despite carrying out the scheduled repairs during operation, there are occasional critical failures of structural elements, leading to the impossibility of their further operation. This requires immediate unscheduled repair or replacement of failed elements. Such critical failures of random structural elements of the technical item under consideration are random events and the time moments of their occurrence are random variables. Therefore, taking into account the impact of such failures on the kinetics of reliability indicators requires the use of simulation modeling of the operation of a technical object. However, this question is beyond the scope of this study and its solution is a promising direction for further research.

An effective approach to ensuring the safe operation of a technical item in the event of possible critical failures is the proactive replacement of structural elements that are subject to such failures at the time of scheduled repairs of the mobile ropeway. The criterion for the need to repair or replace a potentially dangerous element at a time $\tau_{r, i}$ is the achievement of an arbitrary element of the maximum permissible value of the probability of failure:

$1-P_{h, m}\left(\tau_{r, i}\right) \geq\left[Q_{h, m}\right]$

$1-P_{m, m}\left(\tau_{r, i}\right) \geq\left[Q_{m, m}\right]$

$1-P_{e, m}\left(\tau_{r, i}\right) \geq\left[Q_{e, m}\right]$.

The values $\left[Q_{v, m}\right]$ are individual for each structural element of the technical item. Their lower values should correspond to the most responsible and expensive elements. Critical failures of such elements can lead to a greater damage both due to the increased duration and due to the increased costs of repairs. Setting small values $\left[Q_{v m}\right]$ will lead to the need for more frequent repairs of structural elements and, thus, to increased labor and material and financial costs, although the amount of technical risk for the technical item will be low. Otherwise, when assigning higher values $\left[Q_{v, m}\right]$, the cost of repair work will decrease, but the amount of technical 
risk will increase. Thus, the reasonable assignment of marginal probabilities $\left[Q_{v, m}\right]$ is an important technical task and requires an assessment based on technical and economic optimization.

Taking into account the fact of repair of a number of structural elements with critical failures during the scheduled repair $\tau_{r, i}$ requires identification of those elements that meet the criteria In Equations (27) (29). After that, it is necessary to adjust the vectors of the initial conditions for further integration of the differential Equations (1) - (7) for the next inter-repair period $\tau \in\left(\tau_{r, i} ; \tau_{r, i+1}\right)$. The adjustment is performed similarly to the one discussed above in relation to accounting for repairs of structural elements that lead to the non-critical failures. Thus, the first elements of the new initial conditions vectors take the following values:

$$
\begin{aligned}
& P_{0}\left(\tau_{r, i}+0\right)=P_{0}\left(\tau_{r, i}\right)+\sum_{m=1}^{m=n h 1, r, i} P_{h, m}\left(\tau_{r, i}\right)+ \\
& +\sum_{m=1}^{m=n_{m 1, r, i}} P_{m, m}\left(\tau_{r, i}\right)+\sum_{m=1}^{m=n e 1, r, i} P_{e, m}\left(\tau_{r, i}\right) ; \\
& P_{h, 0}\left(\tau_{r, i}+0\right)=P_{h, 0}\left(\tau_{r, i}\right)+\sum_{m=1}^{m=n_{h 1, r, i}} P_{h, m}\left(\tau_{r, i}\right) ; \\
& P_{m, 0}\left(\tau_{r, i}+0\right)=P_{m, 0}\left(\tau_{r, i}\right)+\sum_{m=1}^{m=n_{m 1, r, i}} P_{m, m}\left(\tau_{r, i}\right) ; \\
& P_{e, 0}\left(\tau_{r, i}+0\right)=P_{e, 0}\left(\tau_{r, i}\right)+\sum_{m=1}^{m=n_{e 1}, r i} P_{e, m}\left(\tau_{r, i}\right) .
\end{aligned}
$$

The elements of the new initial condition vectors that correspond to the repaired structural elements of the subsystems are reset to zeroand the remaining elements retain their values.

The graphs of changes in the time probability of finding a mobile ropeway, individual self-propelled rope units and their subsystems in operable state are similar to the graph in Figure 6.

\section{Computer implementation of the mathematical model and analysis of the calculation results}

The developed method of predicting the reliability kinetics indicators of a mobile ropeway, a self-propelled rope unit installation and individual subsystems was implemented in the form of a computer program "ReabilityRopeWay" [21]. It allows to simulate the kinetics of reliability indicators of a structure that includes no more than 200 structural elements, with a service life of up to 50,000 hours. The integration of the Chapman-Kolmogorov equation system in Equations (1) - (7), which is a key process, is performed by the Runge-Kutta method of the fourth order. The program has an extensive library of quantitative data on the failure rate, restoration rate and costs of a large number of structural elements used in mechanical, hydraulic, pneumatic, electrical and electronic systems of machines and technological equipment.

The result of this program is the simulation of changes in time during the standard service life of a mobile ropeway of the following quantitative characteristics:

- the probabilities of finding the technical items under consideration in an operable state and in possible states corresponding to critical failures of structural elements (taking into account the restoration of those elements that reached the permissible value of the probability of failure before the time of the next scheduled repair);

- the probabilities of finding the technical items under consideration in a good state and in possible states corresponding to non-critical failures of structural elements (taking into account the repair of those individual elements that have exhausted the permissible service life before the time of the next scheduled repair).

A test evaluation of the program application [21] for reliability analysis was carried out in relation to a mobile ropeway with a length of $200 \mathrm{~m}$, which has a hydraulic frequency-throttle control of the speed of cargo movement [10] weighing up to $100 \mathrm{kN}$. The ropeway is formed by the two self-propelled rope units with a height of the end tower of $10 \mathrm{~m}$ (Figure 1). The values of the structural elements failure rate, included in the design of the mobile ropeway, were adopted based on the experimental and reference data [22-24].

Figures 7 and 8 show the kinetic curves $P_{r s}(\tau)$ of the probability of finding a mobile ropeway and a separate self-propelled rope unit in operable state during a given service life with different repair intervals and different maximum permissible values of the probability of failure $\left[Q_{v, v m}\right]$. During the simulation, it was assumed that the duration of the inter-repair periods during the entire service life $\Delta \tau_{r, i}=\tau_{r i+1}-\tau_{r, i}=$ const, the probability $\left[Q_{v, m}\right]=$ const for all the elements of the technical object under study.

Kinetic curves $P_{r s}(\tau)$ have a complex geometric shape. The general pattern of a monotonous decrease in probability $P_{r s}$ over the course of operation is superimposed by periodic abrupt changes in the probability value $P_{r s}$ at the time of repairs. Given the complex temporal nature of the kinetic curves, it is advisable to use the integral characteristics of these curves to quantify the influence of various parameters on the reliability indicators of the technical items under consideration. In this study, the following integral characteristics were considered:

- the average probability of finding a technical item in operable state during the entire service life

$\bar{P}_{r s}=T_{l}^{-1} \int_{0}^{T_{l}} P_{r s}(\tau) d \tau$

- the average square deviation of the probability of finding a technical item in operable state during the entire service life 

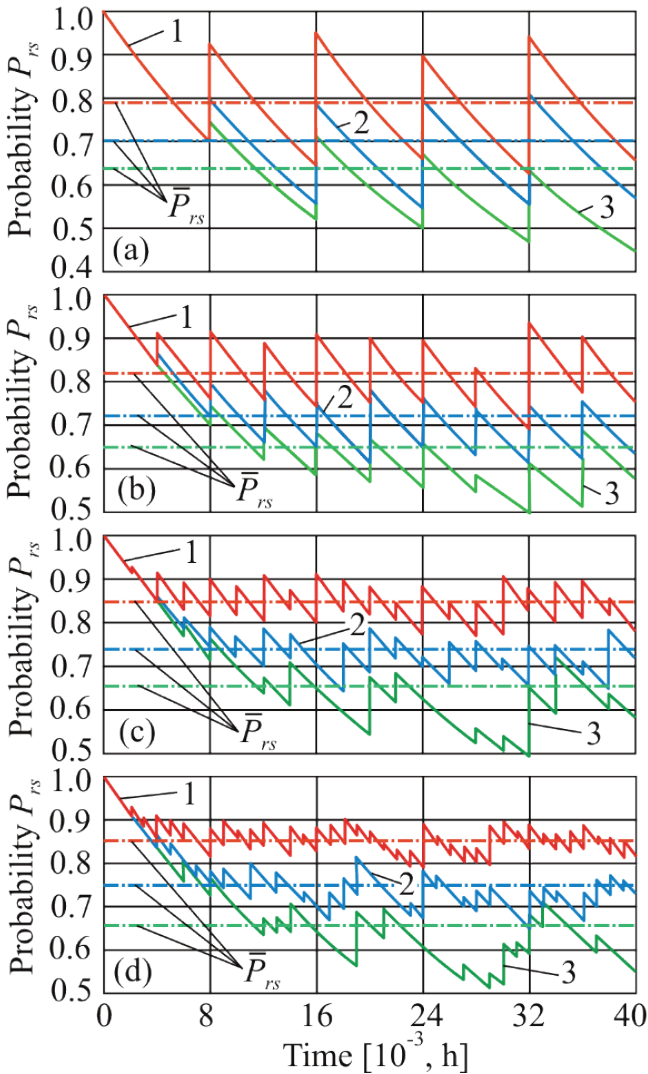

Figure 7 Change in probability of the failure-free operation of a mobile ropeway with different repair intervals: a) $8000 h$; b) $4000 h$; c) $2000 h$;

d) $1000 h\left(1-\left[Q_{v, m}\right]=0.01 ; 2-\left[Q_{v, m}\right]=0.02 ; 3-\left[Q_{v, m}\right]=0.03\right)$

$D_{P, r s}=\left\{T_{l}^{-1} \int_{0}^{T_{l}}\left[P_{r s}(\tau)-\bar{P}_{r s}\right]^{2} d \tau\right\}^{0.5} ;$

- the average value of restoring the probability of finding a technical item in operable state during repairs during the entire service life

$\Delta \bar{P}_{r s}=n_{r}^{-1} \sum_{i=1}^{i=n}\left[P_{r s}\left(\tau_{r, i}+0\right)-P_{r s}\left(\tau_{i}\right)\right] ;$

- the average square deviation of the recovery value of the probability of finding a technical item in operable state during repairs during the entire service life

$D_{\Delta P, r s}=\left\{n_{r}^{-1} \sum_{i=1}^{i=n}\left[P_{r s}\left(\tau_{r, i}+0\right)-P_{r s}\left(\tau_{r, i}\right)\right]^{2}\right\}^{0.5} ;$

- the minimum value of the probability of finding a technical item in operable state during the entire service life

$P_{r s, \min }=\min \left\{\begin{array}{l}P_{r s}\left(\tau_{r, 1}\right) ; P_{r s}\left(\tau_{r, 2}\right) ; \ldots ; P_{r s}\left(\tau_{r, n r}\right) ; \\ P_{r s}\left(T_{l}\right)\end{array}\right\}$

where $n_{r}$ is the total number of repairs during the entire service life.

Replacement or restoration of structural elements, which by the time of the next repair, the probability of failure-free operation $P_{v, m}$ reaches the minimum
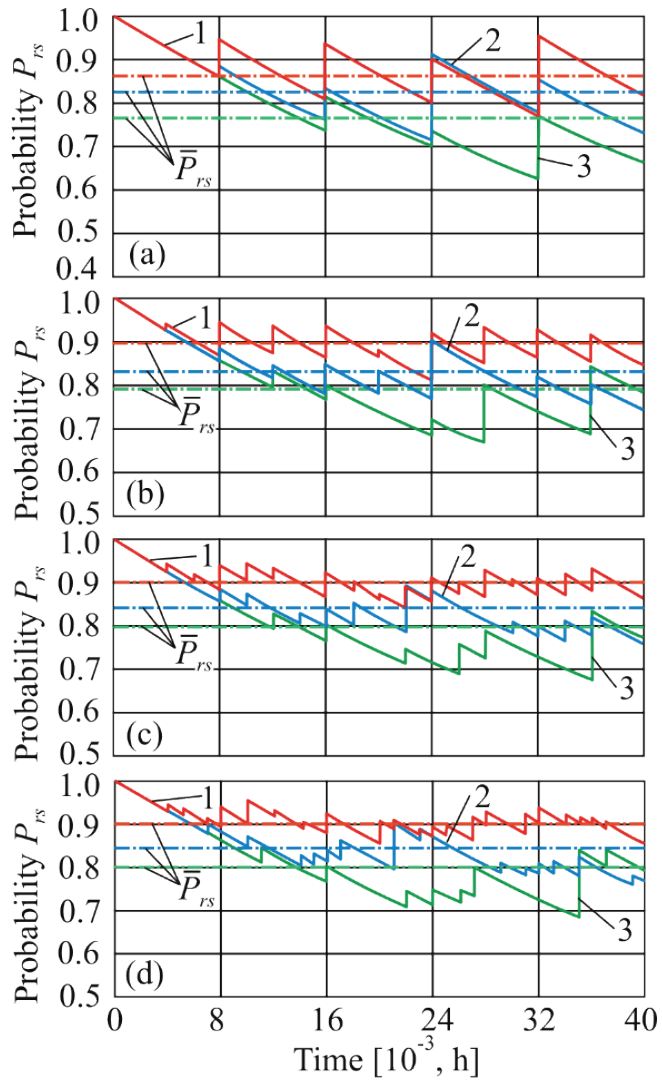

Figure 8 Change in probability of the failure-free operation of a self-propelled rope unit at different repair intervals: a) $8000 h$; b) $4000 h$; c) $2000 h$;

d) $1000 h\left(1-\left[Q_{v, m}\right]=0.01 ; 2-\left[Q_{v, m}\right]=0.02 ; 3-\left[Q_{v, m}\right]=0.03\right)$

allowable value $1-\left[Q_{v, m}\right]$, allows to periodically increase the probability of failure-free operation of the technical item and, thus, effectively resist the objective trend of a monotonous decrease in its reliability as it is used (curve 1 in Figure 6). The graphs in Figures 7 and 8 show that the decrease in the probability $P_{r s}(\tau)$ from 1 to $\sim \bar{P}_{r s}$ occurs in the initial period of operation time (within the time interval from 6000 to 8000 hours). Then, a natural decrease in the probability $P_{r s}(\tau)$ is observed only in the inter-repair period of operation between the two consecutive repairs at $\tau \in\left(\tau_{r, i} ; \tau_{r, i+1}\right)$ (curve 2 in Figure 6). However, the average probability of finding a technical item in operable state $\bar{P}_{r s}$ is maintained almost constant until the end of the service life of a mobile ropeway and a self-propelled rope unit. Thus, the effectiveness of repair measures can be quantified by the amount $\bar{P}_{r s}$ achieved as a result of their implementation. The higher the value $\bar{P}_{r s}$, the more effective the repair measures are to improve the reliability of operation of the technical items under consideration.

Analysis of kinetic curves $P_{r s}(\tau)$ shows that their configuration and quantitative characteristics (including $\left.\bar{P}_{r s}\right)$ are largely determined by parameters such as $\Delta \tau_{r, i}$ and $\left[Q_{v, m}\right]$. Figure 9 shows the influence of parameters $\Delta \tau_{r, i}$ and $\left[Q_{v, m}\right]$ on the integral characteristics of the kinetic curves of a mobile ropeway 
and a self-propelled rope unit. In the entire investigated range of parameters $\Delta \tau_{r i} \in[1000 ; 8000]$ hours (i.e. $\left.n_{r} \in[5 ; 40]\right)$ and $\left[Q_{v, m}\right] \in[0.01 ; 0.05]$, the integral characteristics $\bar{P}_{r s}$ and $P_{r s \text { min }}$ for a mobile ropeway are also significantly lower than the similar characteristics of a rope unit. The quantitative discrepancy increases with an increase in the inter-repair period $\Delta \tau_{r, i}$ (i.e. with a decrease in the total number of repairs $n_{r}$ ) and the maximum permissible probability of critical failure $\left[Q_{v, m}\right]$. Other investigated integral characteristics $D_{P, r s}, \Delta$ $\bar{P}_{r s}$ and $D_{\Delta P, r s}$, on the contrary, are higher than that of a self-propelled rope unit. The quantitative discrepancy between these characteristics increases with increasing $\Delta \tau_{r, i}$ and decreasing $\left[Q_{v, m}\right]$. This correlation between the reliability indicators of a mobile ropeway and a separate self-propelled unit is explained by the fact that the technical devices of the ropeway include a larger number of structural elements with critical failures than the rope unit. This is clearly seen in analysis of the structure of the vectors $\left\{\omega_{h \lambda}\right\},\left\{\omega_{m \lambda}\right\}$ and $\left\{\omega_{e \lambda}\right\}$, forming the matrix in Equation (3).

Figure 9a allows to evaluate the favorable values

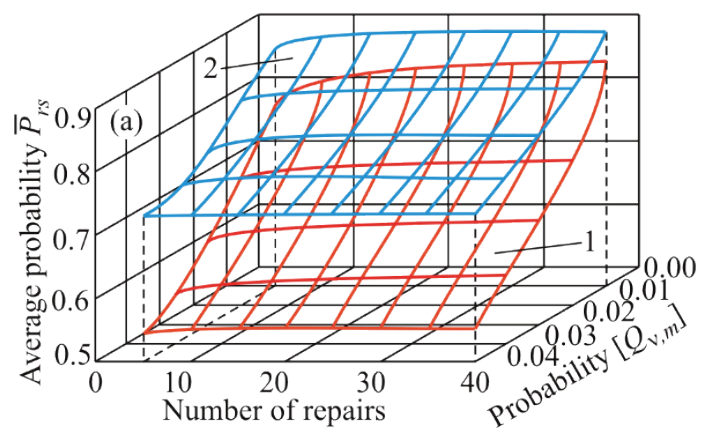

of parameters $\Delta \tau_{r, i}$ and $\left[Q_{v, m}\right]$, using which the level of reliability of mobile ropeway and self-propelled rope unit will have an acceptable value during the entire service life. They are: the duration of the inter-repair period $\Delta \tau_{r, i}$ - no more than 4000 hours, the maximum permissible value of the probability of failure of elements $\left[Q_{v, m}\right]$ - no more than 0.02 .

The presented results of calculation of the mobile ropeway are obtained for the case when only one of the two self-propelled rope units constantly performs the functions of the drive unit during the entire service life to ensure the operation of the carrying-traction rope movement mechanism. Obviously, the alternate use of both self-propelled units as drive units should contribute to a certain increase in the level of reliability of the mobile ropeway. This is due to the fact that those structural elements of the self-propelled unit that do not participate in the operation of the carrying-traction rope movement mechanism do not experience a damaging effect from operational loads and have reduced values of the failure rate. Such structural elements include all the elements with critical failure of the mechanical

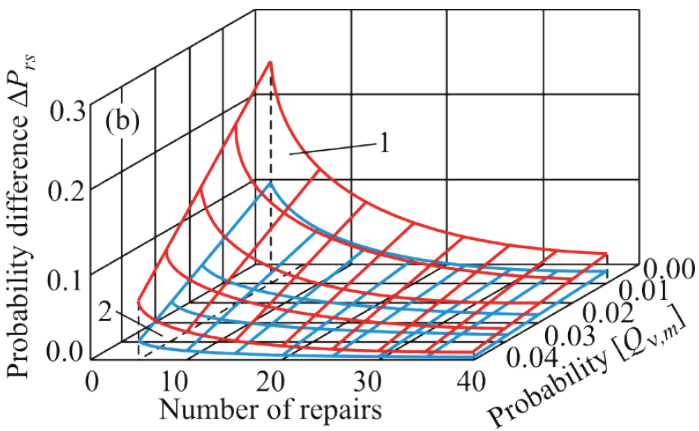

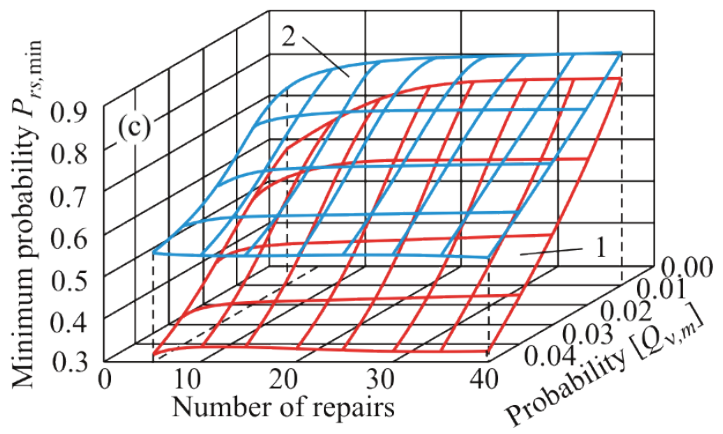

Figure 9 Influence of parameters $\Delta \tau_{r, i}$ and $\left[Q_{v, m}\right]$ on the integral characteristics of the kinetic curves of the ropeway (1) and the self-propelled unit (2): a) $\bar{P}_{r s}$; b) $\Delta \bar{P}_{r s}$; c) $P_{r s, \min }$

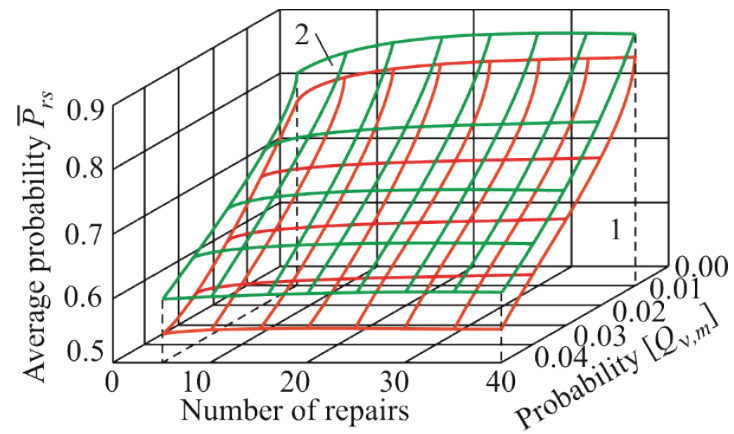

Figure 10 Influence of parameters $\Delta \tau_{r, i}$ and $\left[Q_{v, m}\right]$ on the average probability when operating a mobile ropeway with constant (1) and alternate (2) operation of self-propelled units as drive units 
subsystem and a number of elements of the hydraulic and information-control subsystems. Figure 10 shows the effect of alternating the use of self-propelled rope units as drive units on increasing the average probability of finding a mobile ropeway in operable state. It can be seen that when normalizing the maximum permissible value of the probability of failure of elements in the range of $\left[Q_{v, m}\right]$ $\in 0.015$, the level of reliability of the mobile ropeway will be close (although slightly lower) to the level of reliability of a separate self-propelled unit, regardless of the number of scheduled repairs during the entire service life.

\section{Conclusions}

The approach proposed in this paper allows to predict the kinetics of quantitative indicators of the reliability of mobile ropeways and to ensure the required level of reliability based on the proactive replacement during the scheduled repairs of those structural elements that have reached the maximum permissible value of the probability of a critical failure. This approach is advisable to use at the design stage of the self-propelled rope units in order to predict the reliability and conduct a risk analysis of operation of the mobile ropeways formed with the help of these units.

Computer-based forecasting of the reliability indicators' kinetics allows to solve a number of important technical and economic problems related to the further operation of designed machines and mobile cable cars:

- estimate the maximum reduction in the probability of failure-free operation during the service life of the self-propelled rope units with a given schedule of their repairs;

- to assess the impact of the periodicity of scheduled repairs on reduction of reliability of the self-propelled rope units and their individual subsystems in order to ensure the required level of probability of failurefree operation;

- evaluate the effectiveness of periodic replacement or restoration of the structural elements of the mobile ropeway as a whole and individual self-propelled rope units;

- determine for each scheduled repair the amount of necessary restoration work and the list of structural elements to be restored that lead to critical failures;

- to obtain initial data for calculating and optimizing the technical risk of mobile ropeway operation in accordance with the known approaches [12, 14, 16].

\section{Acknowledgements}

The study was supported by President Grant for Government Support of Young Russian Scientists No. MD-422.2020.8.

\begin{tabular}{|c|c|}
\hline Nomencla & \\
\hline Abbreviation & Meaning/phrase \\
\hline$\overline{n_{r}}$ & The total number of repairs during the entire service life \\
\hline$n_{v 1}\left(n_{v 2}\right)$ & The number of $v$-th subsystem elements leading to a critical (non-critical) failure \\
\hline$n_{v 1 r}\left(n_{v 2 r}\right)$ & $\begin{array}{l}\text { The number of elements of the } v \text {-th subsystem of the rope system leading to a critical (non-critical) } \\
\text { failure }\end{array}$ \\
\hline$n_{v 1, r, i}$ & $\begin{array}{l}\text { The number of elements with critical failures of the } v \text {-th subsystem that were restored at the time of } \\
\text { operation of the mobile ropeway, corresponding to the time of the scheduled repair } \tau_{r, i}\end{array}$ \\
\hline$n_{v 2, r, 1}$ & $\begin{array}{l}\text { The number of elements of the } v \text {-th subsystem that were scheduled repaired at the time of operation of } \\
\text { the mobile ropeway, corresponding to the time of scheduled repair } \tau_{r, 1}\end{array}$ \\
\hline$N_{1}\left(N_{2}\right)$ & The number of elements leading to a critical (non-critical) failure \\
\hline$P_{0}\left(U_{0}\right)$ & The probability of finding a technical item in anoperable (good) state \\
\hline$P_{r s}\left(U_{r s}\right)$ & The probability of failure-free (good) operation of a technical item \\
\hline$P_{v, 0}\left(U_{v, 0}\right)$ & The probability of finding the $v$-th subsystem in an operable (good) state \\
\hline$P_{v}\left(U_{v}\right)$ & The probability of failure-free (good) operation of $v$-th subsystem \\
\hline$Q_{r s}\left(V_{r s}\right)$ & The probability of finding the technical item in an inoperable (faulty) state \\
\hline$Q_{v}\left(V_{v}\right)$ & The probability of finding the $v$-th subsystem in an inoperable (faulty) state \\
\hline$\left[Q_{v, m}\right]$ & $\begin{array}{l}\text { The maximum permissible value of the probability of critical failure of the } m \text {-th element of the } v \text {-th } \\
\text { subsystem }\end{array}$ \\
\hline$\Delta T_{v, m}$ & The repair periodicity of the $m$-th element of the $v$-th subsystem \\
\hline$\Delta \tau_{r, i}$ & The duration of the inter-repair periods during the entire service life \\
\hline$\lambda_{v 1, m}\left(\lambda_{v 2, m}\right)$ & The failure rate of the $m$-th element of the $v$-th subsystem, leading to a critical (non-critical) failure \\
\hline $\begin{array}{l}\mu_{v 1, m}\left(\mu_{v 2, m}\right) \\
\tau_{r, n} \\
\text { subscripts: }\end{array}$ & $\begin{array}{l}\text { The restoration rate of the } m \text {-th element of the } v \text {-th subsystem, leading to a critical (non-critical) failure } \\
\text { The moment of } n \text {-th scheduled repair }\end{array}$ \\
\hline$e$ & Electrical subsystem \\
\hline$h$ & Hydraulic subsystem \\
\hline$m$ & Mechanical subsystem \\
\hline
\end{tabular}




\section{References}

[1] LAGEREV, A. V., LAGEREV, I. A. Designing supporting structures of passenger ropeways of minimum cost based on modular intermediate towers of discretely variable height. Urban Rail Transit [online]. 2020, 6(4), p. 265-277. ISSN 2199-6687. Available from: https://doi.org/10.1007/s40864-020-00137-0

[2] VISSER, R., OBI O. F. Automation and robotics in forest harvesting operations: identifying near-term opportunities. Croatian Journal of Forest Engineering [online]. 2017, 42(1), p. 13-24. ISSN 1845-5719. Available from: https://doi.org/10.5552/crojfe.2021.739

[3] DUVILLARD, P.-A., RAVANEL, L., MARCER, M., SCHOENEICH, P. Recent evolution of damage to infrastructure on permafrost in the French Alps. Regional Environmental Change [online]. 2019, 19(5), p. 1281-1293. ISSN 1436-378X. Available from: https://doi.org/10.1007/s10113-019-01465-z

[4] LAGEREV, A. V., LAGEREV, I. A., TARICHKO, V. I. Impact of design capacity on optimal parameters of freight aerial mono-cable cableways. IOP Conference Series: Earth and Environmental Science[online]. 2019, 378(1), 012063. ISSN 1755-1315. Available from: https://doi.org/10.1088/1755-1315/378/1/012063

[5] HOFFMANN, K. Recent developments in cable-drawn urban transport systems. FME Transactions. 2006, 34, p. 205-212. ISSN 1573-8736.

[6] Guide to managing risks in cable logging. Canberra: Safe Work Australia, 2013. ISBN 978-1-74361-362-7.

[7] Special machines - Valentini Teleferiche [online] [accessed 2021-06-04]. Available from: https://www.valentiniteleferiche.it/en/soluzioni/special-machines

[8] TAJFUN mobile tower yarders [online] [accessed 2021-06-02]. Available from: https://tajfun.com/moz/index.html

[9] PESTAL, E. Ropeways and rope cranes for the transportation of wood and materials / Seilbahnen und Seilkrane in Holz und Materialtransport (in German). Wien: Fromme, 1961.

[10] LAGEREV, A. V., LAGEREV, I. A., TARICHKO, V. I. Modeling of hydrodynamic and kinematic processes during the operation of a mobile cargo rope complex. Journal of Physics: Conference Series [online]. 2021, 1753, 012022. ISSN 1742-6596. Available from: https://doi.org/10.1088/1742-6596/1753/1/012022

[11] MARTINOD, R., ESTEPA, D., PARIS, C., TRUJILLO, A., PINEDA, F., CASTANEDA, L., RESTREPO, J. Journey safety assessment to urban aerial ropeways transport systems based on continuous inspection during operation. Journal of Transportation Safety and Security [online]. 2015, 7(4), p. 279-290. ISSN 1943-9970. Available from: https://doi.org/10.1080/19439962.2014.942018.

[12] TEZAK, S., TOS, Z. Reliability analysis of operation for cableways by FTA (fault tree analysis) method. Promet - Traffic and Transportation [online]. 2010, 22(3), p. 163-173. ISSN 1848-4069. Available from: https://doi.org/10.7307/ptt.v22i3.272

[13] NIKOLIC, R., ARSIC, D., ARSIC, A., SARKOCEVIC, Z., CVETKOVIC, D., HADZIMA, B. The fault tree analysis of causes of the welded pipes in exploitation. Communications - Scientific Letters of the University of Zilina [online]. 2020, 22(1), p. 62-70. ISSN 1335-4205, eISSN 2585-7878. Available from: https://oi.org/10.26552/ com.C.2020.1.62-70

[14] ROSS, S. M. Introduction to probability models. New York, NY: Academic Press, 2014. ISBN 978-0-12-375686-2.

[15] LAGEREV, I. A., TARICHKO, V. I., LAGEREV, A. V. Simulation of the change in the reliability of rope system motion mechanism in mobile ropeway complex. In: 6th International Conference on Industrial Engineering: proceeding. Vol. II. 2020. ISBN 978-1-84800-985-1, p. 745-754.

[16] FINKELSTEIN, M. Failure rate modelling for reliability and risk. In: Springer Series in Reliability Engineering. London: Springer-Verlag London Limited, 2008. ISBN 978-1-84800-986-8.

[17] PatentRU 200827 [online] [accessed 2021-06-02]. Available from: https://www1.fips.ru/registers-doc-view/fips_ servlet

[18] LAGEREV, A. V., LAGEREV, I. A. Reducing labor intensity when computing optimal technical characteristics of aerial ropeways. FME Transactions [online]. 2021, 49(1), p. 72-77. ISSN 1573-8736. Available from: https:// doi.org/10.5937/fme2101072L

[19] LAGEREV, I. A., TARICHKO, V. I., LAGEREV, A. V. Formation of the strategy for restoring the rope system of the mobile transport and reloading rope complex during operation. Nauchno-Tekhnicheskiy Vestnik Bryanskogo Gosudarstvennogo Universiteta [online]. 2020, 2, p. 276-293. ISSN 2413-9920. Available from: https://doi. org/10.22281/2413-9920-2020-06-02-276-293

[20] LAGEREV, A. V., LAGEREV, I. A. Research of dynamics of hydraulic loader crane in case of conjoint movement of sections. In: 4th International Conference on Industrial Engineering: proceeding. 2018. ISBN 978-3-319-95630-5, p. 2283-2294.

[21] The computer program RU 2020615868. Modeling the kinetics of reliability indicators of the mechanism of movement of the rope system of a mobile transport and reloading rope complex [online] [accessed 2021-06-02]. Available from: https://www1.fips.ru/registers-doc-view/fips_servlet 
[22] Failure rate of elements: reference guide [online] [accessed 2021-06-02]. Available from: https://areliability.com/ intensivnost-otkazov-elementov-spravochnik/

[23] DENSON, W., CHANDLER, G., CROWELL, W., WANNER, R. Nonelectronic parts reliability data. Chicago, IL: IIT Research Institute, 1991.

[24] Electronic reliability design handbook (MIL-HDBK-338B). New York, NY: Air Force Research Laboratory Information, 1999. 